\title{
Cardiac sarcoidosis and ventricular arrhythmias. A rare association of a rare disease. A retrospective cohort study from the National Inpatient Sample and current evidence for management
}

\author{
Amr Salama ${ }^{1}$, Abdullah Abdullah ${ }^{1}$, Abdul Wahab ${ }^{1}$, George Eigbire ${ }^{1}$, \\ Ryan Hoefen ${ }^{1,2,4}$, Richard Alweis ${ }^{1,2,3}$ \\ ${ }^{1}$ Department of Medicine, Unity Hospital, Rochester, New York, United States \\ ${ }^{2}$ Department of Medicine, University of Rochester School of Medicine and Dentistry, \\ Rochester, New York, United States \\ ${ }^{3}$ School of Health Sciences, Rochester Institute of Technology, New York, United States \\ ${ }^{4}$ Department of Cardiology, Rochester Regional Health, New York, United States
}

\begin{abstract}
Background: Sarcoidosis is an increasingly recognized multi-systemic condition. Cardiac sarcoidosis is associated with ventricular arrhythmias and higher mortality rates. Little epidemiological data is available regarding the disease and associated ventricular arrhythmias.

Methods: Data from the National Inpatient Sample (NIS) database 2012-2014, were reviewed. Discharges associated with sarcoidosis were identified as the target population using relevant ICD-9-CM codes. Primary outcome was a diagnosis of ventricular tachycardia (VT) in the sarcoidosis population. Secondary outcomes include rate of ventricular fibrillation (VF) and cardiac arrest. Subgroup analyses were performed to examine the association of VT with multiple potential confounding clinical variables. Results: Of 18,013,878 health encounters, 46,289 (0.26\%) subjects had a diagnosis of sarcoidosis. VT and VF were more prevalent among patients with sarcoidosis compared to those without a diagnosis of sarcoidosis (2.29\% vs. $1.22 \%$; $p<0.001$ and $0.25 \%$ vs. $0.21 \% ; p<0.001$, respectively). Sarcoidosis was also associated with a higher prevalence of cardiac arrest $(0.72 \%$ vs. $0.6 \% ; p<0.001)$. In unadjusted analyses, all examined comorbidities were significantly more common in those with sarcoidosis, including diabetes mellitus (31.6\% vs. 21.25\%; $p<0.001$ ), hypertension (65.2\% vs. $51.74 \%$; $p<0.001)$, chronic kidney disease (21.09\% vs. $14.02 \%$; $p<0.001)$, heart failure $(24.87 \%$ vs. $15 \%$; $p<0.001)$ and acute coronary syndrome $(4.32 \%$ vs. $3.35 \%$; $p<0.001)$.

Conclusions: The present study showed that sarcoidosis was associated with increased rates of ventricular tachyarrhythmia, which can affect the overall disease morbidity and mortality. (Cardiol J 2020; 27, 3: 272-277)

Key words: cardiac sarcoidosis, ventricular tachycardia, ventricular fibrillation, cardiac death
\end{abstract}

Address for correspondence: Amr Salama, MD, Department of Medicine, Unity Hospital, 1555 Long Pond Road, Rochester, New York, USA, 14626, tel: +1 5852982834, e-mail: Amr.salama@rochesterregional.org 


\section{Introduction}

Sarcoidosis is a multi-system granulomatous disease of unclear etiology with variable presentation. The prevalence ranges from 10.9 per 100,000 for whites to 35.5 per 100,000 for blacks [1]. The pathological hallmark of the disease is non-caseating granulomas. Cardiac sarcoidosis (CS) is increasingly recognized and associated with higher morbidity and mortality rates. Studies show a wide range (3.7-54.9\%) of cardiac involvement in patients with systemic sarcoidosis [2].

Conduction abnormalities and arrhythmias are the most common clinical manifestations of $\mathrm{CS}$, followed by congestive heart failure (HF) and sudden death [3]. The prevalence of specific arrhythmias is as follows: $26-62 \%$ atrioventricular block, $0-15 \%$ supraventricular tachycardia, $2-42 \%$ ventricular tachycardia (VT), and $12-65 \%$ sudden cardiac death [4]. Given the very rare nature of CS, electrophysiologists rely on small observational studies. It is hypothesized herein, that CS could be a significant independent predictor for development of VT. The National Inpatient Sample (NIS) was analyzed to test this possibility.

\section{Methods}

\section{Study design and data source}

Data from the NIS database, part of the Healthcare Cost and Utilization Project (HCUP), an Agency for Healthcare Research and Quality (AHRQ) was used. This is the largest inpatient healthcare database available to the public in the United States of America. The study utilized data for the years from 2012 to 2014 . The NIS includes a 20-percent stratified sample of all discharges from United States hospitals participating in the HCUP, 7-8 million discharges per year, excluding rehabilitation and long-term acute care hospitals. It contains variables extracted from hospital discharge records, including demographic characteristics, principal diagnosis, secondary diagnoses or comorbidities, insurance, cost and the procedures performed. The database allows for calculating national estimates by providing a "weight" variable [5]. When the data expands to estimate nation-wide discharges, it provides estimates corresponding to about 38 million annual hospitalizations or $97 \%$ of discharges nationwide [6]. Estimates from the NIS have been validated against the Centers for Medicare and Medicaid Services registry [5, 7]. Each discharge record was treated as an individual database entry. The databases used International
Classification of Diseases-9th Edition (ICD-9) codes to identify diagnoses and procedures.

\section{Subjects and variables}

The study population was derived from $18,013,878$ hospital discharges available in the NIS. Patients with VT and sarcoidosis were identified using ICD-9 codes, 427.1 and 135, respectively. Primary outcome was a diagnosis of VT in the sarcoidosis population. Secondary outcomes include rate of ventricular fibrillation (VF) and cardiac arrest. Analyses of clinical variables were performed to examine the association of VT with multiple confounding factors.

Confounding variables were identified using the ICD-9 diagnosis codes including both patient and hospital level variables, in addition to data year. Patient characteristics included age, gender, diabetes mellitus, HF, hypertension, acute coronary syndrome (ACS) (during the same hospitalization) and chronic kidney disease.

\section{Statistical analysis}

Normally distributed continuous variables were expressed as means \pm standard deviation and compared using the Student t-test. Continuous variables that were not normally distributed were expressed as medians with interquartile ranges and compared using Mann-Whitney test. Categorical variables were compared using the $\chi^{2}$ test. In order to adjust for potential confounders, multivariable logistic regression was performed.

A median Charlson-Deyo Index (CDI) was calculated for both sarcoidosis and non-sarcoidosis groups. CDI is a validated measure of comorbidities for administrative data [8]. All analyses were performed using STATA 2015 TX: Stata Corp LP. Two-tailed $p$-value $<0.05$ was considered statistically significant.

\section{Results}

$18,013,878$ health encounters were identified from the NIS that represent hospitalizations from 2012 to 2014 . Of these, $46,289(0.26 \%)$ subjects had a diagnosis of sarcoidosis, and the remaining $17,967,589(99.7 \%)$ do not have sarcoidosis among their discharge diagnoses. Baseline characteristics of patients with and without sarcoidosis diagnosis are shown in Table 1.

In unadjusted analyses, patients with sarcoidosis were middle-aged (mean $58.2 \pm 13.6$ years) and predominantly female (64.2\%). All examined comorbidities were significantly more common in 
Table 1. Baseline variables between target group (sarcoidosis patients) and control group.

\begin{tabular}{lccc}
\hline Variables & $\begin{array}{c}\text { Sarcoidosis } \\
\text { (n= 46,289) }\end{array}$ & $\begin{array}{c}\text { No sarcoidosis } \\
\text { (n= 17,967,589) }\end{array}$ & P \\
\hline Age [years] & $58.2 \pm 13.6$ & $57.3 \pm 20.6$ & $<0.001$ \\
Male & $35.8 \%(16,556)$ & $40.9 \%(7,352,675)$ & $<0.001$ \\
Diabetes mellitus & $31.60 \%(14,626)$ & $21.25 \%(3,818,578)$ & $<0.001$ \\
Hypertension & $65.20 \%(30,179)$ & $51.74 \%(9,297,154)$ & $<0.001$ \\
Chronic kidney disease & $21.09 \%(9,763)$ & $14.02 \%(2,519,784)$ & $<0.001$ \\
Acute coronary syndrome & $3.35 \%(1,551)$ & $4.32 \%(777,054)$ & $<0.001$ \\
Heart failure & $24.87 \%(11,510)$ & $15.01 \%(2,696,906)$ & $<0.001$ \\
Charlson-Deyo Index & $2.3 \pm 2.07$ & $1.7 \pm 2.15$ & $<0.001$ \\
Ventricular tachycardia & $2.29 \%(1,059)$ & $1.22 \%(218,330)$ & $<0.001$ \\
Ventricular fibrillation & $0.25 \%(118)$ & $0.21 \%(38,012)$ & $<0.001$ \\
Cardiac arrest & $0.72 \%(331)$ & $0.60 \%(108,312)$ & $<0.001$ \\
\hline
\end{tabular}

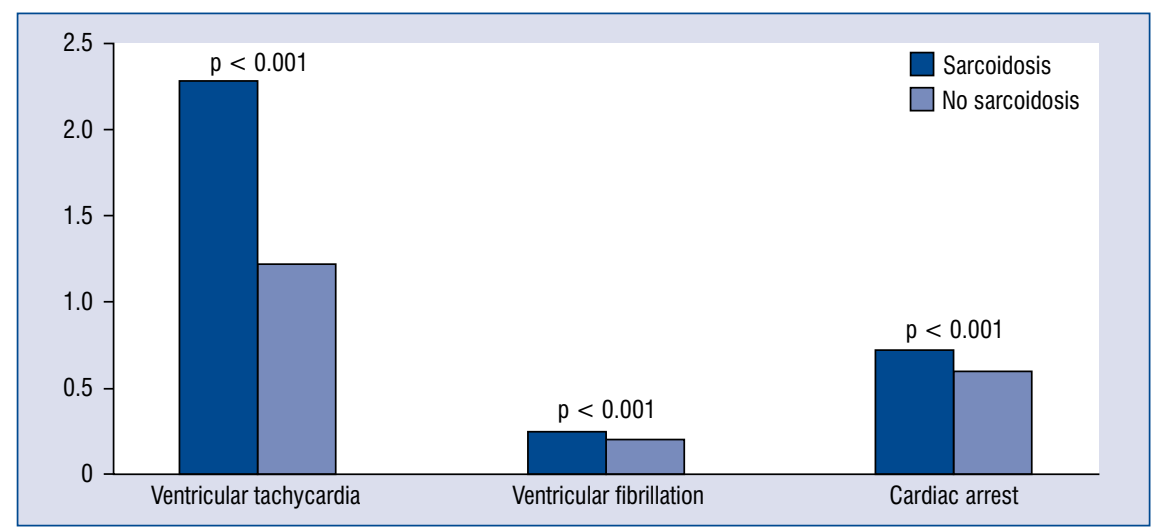

Figure 1. Summaries of outcomes in patients with and without sarcoidosis.

those with sarcoidosis, including diabetes mellitus (31.6\% vs. $21.25 \%$; $<<0.001)$, hypertension $(65.2 \%$ vs. $51.74 \% ; \mathrm{p}<0.001)$, chronic kidney disease $(21.09 \%$ vs. $14.02 \%$; $p<0.001)$, HF $(24.87 \%$ vs. $15 \% ; \mathrm{p}<0.001)$ and $\operatorname{ACS}(4.32 \%$ vs. $3.35 \%$; $\mathrm{p}<0.001)$.

The CDI was 2.3 in the sarcoidosis group vs. 1.7 in the control group. $2.29 \%(1,059)$ of patients in the sarcoidosis group had a diagnosis of VT vs. $1.22 \%$ $(218,330)$ of the control group, $\mathrm{p}<0.001$ (Fig. 1$)$.

The adjusted odds ratio (OR) of VT in the sarcoidosis group was 1.83 (95\% confidence interval [CI] 1.72-1.95; p < 0.001). Table 2 shows independent predictors of VT among CS patients in a multivariable analysis. HF (OR 5.24; $95 \% \mathrm{CI}$ 5.19-5.29; $\mathrm{p}<0.001)$ and ACS (OR 2.84; 95\% CI $2.81-2.87 ; \mathrm{p}<0.001)$ were associated with higher OR for VT.

Ventricular fibrillation was also more common in the sarcoidosis group $(0.25 \%$ vs. $0.21 \%$; $\mathrm{p}<0.001)$. The OR of VF in the sarcoidosis group after multivariable logistic regression analysis was 1.24 (95\% CI 1.03-1.49; p < 0.019; Table 3). Rates of VF was higher with ACS (OR 10.45; 95\% CI 10.22-10.68; $\mathrm{p}<0.001$ ) and HF (OR 3.58; 95\% CI 3.50-3.67; $\mathrm{p}<0.001)$.

Finally, sarcoidosis carries a higher prevalence of cardiac arrest $(0.72 \%$ vs. $0.6 \%$; $<<0.001)$. Cardiac arrest remained more prevalent in sarcoidosis patients (OR 1.21; 95\% CI 1.08-1.35; p < 0.001) as well as ACS 4.91 (95\% CI 4.84-4.98; $\mathrm{p}<0.001)$ and HF (OR 2.14; 95\% CI 2.11-2.17; $\mathrm{p}<0.001$ ) in multivariable logistic regression analysis as shown in Table 4.

\section{Discussion}

Cardiac involvement in sarcoidosis is associated with increased morbidity and mortality. Isolated CS is much more common than suspected; 
Table 2. Independent predictors of ventricular tachycardia.

\begin{tabular}{lccc}
\hline Variables & Odds ratio & $95 \% \mathrm{Cl}$ & $\mathbf{P}$ \\
\hline Age & 1.01 & $1.013-1.014$ & $<0.001$ \\
Female & 0.44 & $0.43-0.44$ & $<0.001$ \\
Sarcoidosis & 1.83 & $1.72-1.95$ & $<0.001$ \\
ACS & 2.84 & $2.81-2.87$ & $<0.001$ \\
Heart failure & 5.24 & $5.19-5.29$ & $<0.001$ \\
\hline
\end{tabular}

ACS - acute coronary syndrome; $\mathrm{Cl}$ - confidence interval

Table 3. Independent predictors of ventricular fibrillation.

\begin{tabular}{lccc}
\hline Variables & Odds ratio & $95 \% \mathrm{Cl}$ & $\mathbf{P}$ \\
\hline Age & 0.998 & $0.997-0.999$ & $<0.001$ \\
Female & 0.44 & $0.43-0.45$ & $<0.001$ \\
Sarcoidosis & 1.24 & $1.03-1.49$ & 0.019 \\
ACS & 10.45 & $10.22-10.68$ & $<0.001$ \\
Heart failure & 3.58 & $3.50-3.67$ & $<0.001$ \\
\hline
\end{tabular}

ACS - acute coronary syndrome; $\mathrm{Cl}$ - confidence interval

Table 4. Independent predictors of cardiac arrest.

\begin{tabular}{lccc}
\hline Variables & Odds ratio & $95 \% \mathrm{Cl}$ & $\mathbf{P}$ \\
\hline Age & 1.01 & $1.013-1.014$ & $<0.001$ \\
Female & 0.65 & $0.65-0.67$ & $<0.001$ \\
Sarcoidosis & 1.21 & $1.08-1.35$ & $<0.001$ \\
ACS & 4.91 & $4.84-4.98$ & $<0.001$ \\
Heart failure & 2.14 & $2.11-2.17$ & $<0.001$ \\
\hline
\end{tabular}

ACS - acute coronary syndrome; $\mathrm{Cl}$ - confidence interval

in a retrospective study, $66 \%$ of patients with CS had disease isolated to the heart [9]. To date, CS has been extremely difficult to diagnose due to non-specific clinical manifestations, and the limited sensitivity and specificity of various diagnostic modalities [10]. There is also a lack of consensus regarding the diagnostic criteria for CS. Ventricular arrhythmias (VT, multifocal or frequent PVCs) are one of the minor criteria of the Japanese Ministry of Health and Welfare Criteria for diagnosis of CS, while the Heart Rhythm Society consensus statement did not include ventricular arrhythmias as a criteria for diagnosing CS [11].

The present study analyzed a large database and revealed an increased rate of VT in patients with sarcoidosis (2.29\%) compared to patients without sarcoidosis $(1.22 \%)$; $<0.001$. HF, hypertension, chronic kidney disease and diabetes mellitus were more common in the sarcoidosis group. Even after adjustment for possible biologi- cal confounders, the odds for having VT was still higher $(1.82$; 95\% CI 1.72-1.95; p < 0.001) in sarcoidosis patients, which suggest that CS may be an independent risk factor for VT.

Other reports suggested VT is the most frequent arrhythmia noted in CS with a reported incidence of $23 \%$ [12]. ACS and $\mathrm{HF}$ are traditional risk factors for development of VT, a fact that was confirmed after multivariable logistic regression analyses that showed higher odds of VT in ACS and HF population $(2.84 ; 95 \%$ CI 2.81-5.87; $\mathrm{p}<0.001$ and 5.24; 95\% CI 5.19-5.29; $\mathrm{p}<0.001$, respectively.

Secondary outcomes of VF and cardiac arrest were both higher in the sarcoidosis group $(0.25 \%$ vs. $0.21 \% ; \mathrm{p}<0.001$ and $0.72 \%$ vs. $0.6 \% ; \mathrm{p}<0.001$, respectively. Moreover, ACS and HF were associated with higher cardiac arrest rates (Table 4). This is consistent with a high mortality rate in CS seen by Roberts et al. [13], where sudden death was the most common manifestation of CS. The 
present analysis shows increased rates for $\mathrm{VF}$ in sarcoidosis patients (OR 1.24; 95\% CI 1.03-1.49; $\mathrm{p}<0.019$; Table 3).

The causality between VT and sarcoidosis cannot be assessed in a retrospective database study such as this one. Postulated mechanisms include active inflammation [11], enhanced automaticity [12], and ventricular activation and recovery process, which explains the reentry mechanism that leads to VT, the most frequent arrhythmia noted in CS [14]. A multicenter registry of patents with CS who underwent an electrophysiology study showed that the mechanism of VT was reentry in virtually all patients [15].

Management of arrhythmias in CS is difficult, and effective control of VT is often not achievable with a single method therapy. Steroids remain central in the management of CS as it can ameliorate cardiac dysfunction [16]. However, recurrent VT may increase with steroid therapy as active granulomas are replaced by fibrosis, a substrate for reentrant arrhythmias [17]. Corticosteroids have been associated with ventricular aneurysm formation [13], so immunosuppressive therapies are recommended in cases of resistant arrhythmias or as a steroid-sparing strategy [18].

Amiodarone and sotalol are widely used to treat VT in patients with $\mathrm{CS}$, although adverse reactions may limit their practicality [15]. Amiodarone use may be limited by the incidence of pulmonary complications which are often difficult to distinguish from pulmonary sarcoidosis. betablockers have been shown to increase the incidence of heart block in patients with CS, thus limiting the utility of sotalol [14].

Prophylactic implantable cardioverter-defibrillator (ICD) placement is recommended for patients with sarcoidosis who develop sustained VT or left ventricular systolic dysfunction with left ventricular ejection fraction $\leq 35 \%$ despite optimal medical therapy and immunosuppression. It may also be a reasonable treatment for patients with $\mathrm{CS}$, regardless of their left ventricular function, after syncope, near syncope, or inducible VT [11]. ICD placement will prevent VT progression but will not prevent recurrence. Therefore, concurrent treatment with anti-arrhythmic therapy is necessary in refractory VT [19]. Some experts recommend ICD placement in patients with sarcoidosis and non-sustained VT given the high rate of recurrent VT despite antiarrhythmic and corticosteroid treatment [10].

Radiofrequency ablation is very effective in decreasing or eliminating VT in patients with CS according to data from a multicenter registry that showed a $98 \%$ reduction in VT burden in the first 3 months after ablation [15]. Resistant ventricular tachyarrhythmia and severe intractable HF, especially in younger patients, are indications for cardiac transplantation in patient with CS [14].

\section{Study power}

According to available research, this is the largest epidemiological study to assess the role of sarcoidosis in VT. Primary analysis confirms an increased rate of VT in sarcoidosis, even after adjustment for possible biological confounders. The strength of these findings lies in the fact that HCUP is a well-validated database that has been previously utilized in similar research studies.

\section{Limitation of the study}

ICD-9 codes were used to identify patient comorbidities. However, prior studies have demonstrated that ICD-9 codes exhibit high (> 90\%) sensitivity, specificity and positive predictive value for cardiovascular disorders such as HF, myocardial infarction and arrhythmias, when compared to full medical chart review [7]. Also, due to the nature of the NIS database analysis, functional outcomes and long-term data were not available. Moreover, specific information regarding medications, electrophysiological studies, or echocardiographic parameters were not available. Lastly, due to the observational nature of the study, and unmeasured confounding factors may have affected interpretation and causality cannot be demonstrated.

\section{Conclusions}

Sarcoidosis carries a risk of various cardiac complications. The present analysis shows that hospitalized patients with sarcoidosis have elevated risk of VT, VF, and cardiac arrest.

\section{Conflict of interest: None declared}

\section{References}

1. Rybicki BA, Major M, Popovich J, et al. Racial differences in sarcoidosis incidence: a 5-year study in a health maintenance organization. Am J Epidemiol. 1997; 145(3): 234-241, doi: 10.1093/ /oxfordjournals.aje.a009096.

2. Nery PB, Leung E, Birnie DH. Arrhythmias in cardiac sarcoidosis: diagnosis and treatment. Curr Opin Cardiol. 2012; 27(2): 181-189, doi: 10.1097/HCO.0b013e32834e4c7c, indexed in Pubmed: 22186166.

3. Hulten E, Aslam S, Osborne M, et al. Cardiac sarcoidosisstate of the art review. Cardiovasc Diagn Ther. 2016; 6(1): 
50-63, doi: 10.3978/j.issn.2223-3652.2015.12.13, indexed in Pubmed: 26885492.

4. Ipek E, Demirelli S, Ermis E, et al. Sarcoidosis and the heart: A review of the literature. Intractable Rare Dis Res. 2015; 4(4): 170-180, doi: 10.5582/irdr.2015.01023, indexed in Pubmed: 26668777.

5. Healthcare Cost and Utilization Project (HCUP). Introduction to the HCUP National Inpatient Sample (NIS) 2014. 2016; 4287: $1-52$.

6. Agency for Healthcare Research and Quality. National Inpatient Sample (NIS) Redesign Final Report. HCUP Methods Ser. 2014; 04: $1-32$.

7. Tamariz L, Harkins T, Nair V. A systematic review of validated methods for identifying ventricular arrhythmias using administrative and claims data. Pharmacoepidemiol Drug Saf. 2012; 21 Suppl 1: 148-153, doi: 10.1002/pds.2340, indexed in Pubmed: 22262601.

8. Charlson ME, Pompei P, Ales KL, et al. A new method of classifying prognostic comorbidity in longitudinal studies: development and validation. J Chronic Dis. 1987; 40(5): 373-383, indexed in Pubmed: 3558716.

9. Lynch, J. P. Cardiac involvement in sarcoidosis: Evolving concepts in diagnosis and treatment. Semin. Respir. Crit. Care Med. 2014; 35: 372-390.

10. Doughan AR, Williams BR. Cardiac sarcoidosis. Heart. 2006; 92(2): 282-288, doi: 10.1136/hrt.2005.080481.

11. Birnie DH, et al. HRS expert consensus statement on the diagnosis and management of arrhythmias associated with cardiac sarcoidosis. Heart Rhythm. 2014; 11: 1305-1324.

12. Mehta D, Lubitz SA, Frankel Z, et al. Cardiac involvement in patients with sarcoidosis: diagnostic and prognostic value of outpatient testing. Chest. 2008; 133(6): 1426-1435, doi: 10.1378/ /chest.07-2784, indexed in Pubmed: 18339784.

13. Roberts WC, McAllister HA, Ferrans VJ. Sarcoidosis of the heart. A clinicopathologic study of 35 necropsy patients (group I) and review of 78 previously described necropsy patients (group II). Am J Med. 1977; 63(1): A81, doi: 10.1016/0002-9343(77)90145-0.

14. Sekhri V, Sanal S, Delorenzo LJ, et al. Cardiac sarcoidosis: a comprehensive review. Arch Med Sci. 2011; 7(4): 546-554, doi: 10.5114/aoms.2011.24118, indexed in Pubmed: 22291785.

15. Jefic D, Joel B, Good E, et al. Role of radiofrequency catheter ablation of ventricular tachycardia in cardiac sarcoidosis: report from a multicenter registry. Heart Rhythm. 2009; 6(2): 189-195, doi: 10.1016/j.hrthm.2008.10.039, indexed in Pubmed: 19187909.

16. Kopriva P, Griva M, Tüdös Z. Management of cardiac sarcoidosis: A practical guide. Cor et Vasa. 2018; 60(2): e155-e164, doi: 10.1016/j.crvasa.2017.05.012.

17. Koplan BA, Soejima K, Baughman K, et al. Refractory ventricular tachycardia secondary to cardiac sarcoid: electrophysiologic characteristics, mapping, and ablation. Heart Rhythm. 2006; 3(8): 924-929, doi: 10.1016/j.hrthm.2006.03.031, indexed in Pubmed: 16876741.

18. Winters S, Cohen M, Greenberg S, et al. Sustained ventricular tachycardia associated with sarcoidosis: Assessment of the underlying cardiac anatomy and the prospective utility of programmed ventricular stimulation, drug therapy and an implantable antitachycardia device. J Am Coll Cardiol. 1991; 18(4): 937-943, doi: 10.1016/0735-1097(91)90750-4.

19. Epstein A, DiMarco J, Ellenbogen K, et al. ACC/AHA/HRS 2008 Guidelines for Device-Based Therapy of Cardiac Rhythm Abnormalities. Heart Rhythm. 2008; 5(6): e1-e62, doi: 10.1016/j. hrthm.2008.04.014. 